OPEN ACCESS

Edited by: Stefano Caserta, University of Hull, United Kingdom

Reviewed by:

Gendie Lash,

Guangzhou Medical University, China

Jelmer R. Prins,

University Medical Center Groningen,

Netherlands

*Correspondence:

Gemma Sullivan

gemma.sullivan@ed.ac.uk

Specialty section: This article was submitted to Inflammation,

a section of the journa

Frontiers in Immunology

Received: 08 June 2021 Accepted: 09 August 2021 Published: 27 August 2021

Citation:

Sullivan G, Galdi P.

Borbye-Lorenzen N, Stoye $D Q$, Lamb GJ, Evans MJ, Skogstrand K, Chandran S and Boardman JP (2021)

Preterm Birth Is Associated With

Immune Dysregulation Which

Persists in Infants Exposed to Histologic Chorioamnionitis.

Front. Immunol. 12:722489.

doi: 10.3389/fimmu.2021.722489

\section{Preterm Birth Is Associated With Immune Dysregulation Which Persists in Infants Exposed to Histologic Chorioamnionitis}

\author{
Gemma Sullivan ${ }^{1 *}$, Paola Galdi ${ }^{1}$, Nis Borbye-Lorenzen ${ }^{2}$, David Q. Stoye ${ }^{1}$, Gillian J. Lamb ${ }^{1}$, \\ Margaret J. Evans ${ }^{3}$, Kristin Skogstrand ${ }^{2}$, Siddharthan Chandran ${ }^{4,5}$ \\ and James P. Boardman ${ }^{1,4}$ \\ ${ }^{1}$ Medical Research Council (MRC) Centre for Reproductive Health, University of Edinburgh, Edinburgh, United Kingdom, \\ ${ }^{2}$ Danish Center for Neonatal Screening, Statens Serum Institut, Copenhagen, Denmark, ${ }^{3}$ Department of Pathology, Royal \\ Infirmary of Edinburgh, Edinburgh, United Kingdom, ${ }^{4}$ Centre for Clinical Brain Sciences, University of Edinburgh, Edinburgh, \\ United Kingdom, ${ }^{5}$ Medical Research Council (MRC) Centre for Regenerative Medicine, University of Edinburgh, Edinburgh, \\ United Kingdom
}

Introduction: Preterm infants are at increased risk of exposure to histologic chorioamnionitis ( $\mathrm{HCA})$ when compared to term-born controls, and this is associated with several neonatal morbidities involving brain, lungs and gut. Preterm infants could benefit from immunomodulatory therapies in the perinatal period, but development of rational treatment strategies requires improved characterization of the perinatal response to HCA. We had two objectives: The first, to characterize the umbilical cord blood immune profile in preterm infants compared to term-born controls; the second, to investigate the postnatal immune response in preterm infants exposed to HCA versus those who were not.

Population: For objective one 59 term infants [mean gestational age (GA) $39^{+4}\left(37^{+3}\right.$ to $\left.42^{+0}\right)$ ] and 55 preterm infants [mean GA29 $9^{+0}\left(23^{+3}\right.$ to $\left.32^{+0}\right)$ ] with umbilical cord samples available were included; for objective two we studied 96 preterm infants [mean GA29+1 $\left(23^{+2}\right.$ to $\left.32^{+0}\right)$ ] for whom placental histology and postnatal blood samples were available.

Methods: Placental histopathology was used to identify reaction patterns indicative of HCA, and a customized immunoassay of 24 inflammatory markers and trophic proteins selected to reflect the perinatal immune response was performed on umbilical cord blood in term and preterm participants and postnatal day 5 blood in the preterm group.

Results: The umbilical cord blood immune profile classified gestational age category with $86 \%$ accuracy (95\% Cl 0.78-0.92), p-value $=1.242 \times 10^{-14}$. Pro-inflammatory proteins IL-6, MCP-1 and CRP were elevated in the cord blood of preterm infants whilst BDNF, C3, C9, IL-18, MMP-9 and RANTES were decreased, compared to infants born at term. 
In preterm infants, exposure to HCA was associated with elevations in 8 immune proteins on postnatal day 5 (BDNF, C3, C5a, C9, IL-8, MCP-1, MIP-1 $\beta$ and MMP-9) when compared to preterm infants who were not exposed.

Conclusion: Preterm birth is associated with a distinct immune profile in umbilical cord blood and preterm infants exposed to HCA with evidence of a fetal inflammatory response have specific alterations in immune function that are apparent on day 5 of postnatal life.

Keywords: complement, cytokine, fetal inflammatory response, inflammation, immunity, interleukin, preterm birth, histologic chorioamnionitis

\section{INTRODUCTION}

Perinatal immune processes have a crucial role in neurodevelopment and early life inflammatory exposures are associated with an increased risk of neuropsychiatric disorders such as autism spectrum disorder, schizophrenia, bipolar disorder and depression $(1,2)$. Preterm infants may be exposed to multiple episodes of perinatal infection/inflammation and are particularly vulnerable to brain injury resulting from a dysregulated immune response during a critical period of CNS development (3).

Preterm infants have a distinct immune profile in umbilical cord blood and cerebrospinal fluid that includes higher levels of pro-inflammatory cytokines and lower levels of growth factors when compared to term-born controls, but there is uncertainty about the extent to which this is influenced by antenatal factors, environmental exposures and/or developmental regulation (46). Histologic chorioamnionitis (HCA), defined as inflammation of the chorioamniotic membranes, is strongly associated with preterm birth $(7,8)$ and increases the risk of neonatal morbidities including lung disease, intraventricular hemorrhage, sepsis and necrotizing enterocolitis (9-14). HCA has also been implicated in the development of white matter injury, cerebral palsy and neurodevelopmental impairment (15-18) and we have previously reported that this may be mediated by a distinct cord blood immune profile in preterm infants (19). When HCA involves a fetal inflammatory response (FIR), these risks appear to be increased further, suggesting that organ injury is mediated by a systemic fetal inflammatory response syndrome (FIRS). FIRS was initially defined using threshold values of IL-6 concentration in umbilical cord blood (20,21), although subsequent studies have shown that histopathological FIR is associated with elevated concentrations of cytokines (IL-1 $\beta$, IL6 and TNF- $\alpha$ ), chemokines (IL-8, MCP-1, MIP-1 $\beta$, RANTES), matrix metalloproteinases (MMP-1 and MMP-9) and CRP (19, 22-26). In some preterm infants, blood concentrations of inflammatory mediators remain elevated for weeks after birth $(27,28)$ and may be associated with higher circulating levels of neurotrophic growth factors (29). However, neurotrophic capability following exposure to intrauterine inflammation is not well understood and previous study designs leave uncertainty about the role of the complement system in perinatal inflammation, which plays a critical role in the innate immune response.
Preterm infants could benefit from immunomodulatory therapies in the perinatal period, but development of rational treatment strategies requires improved characterization of the neonatal immune profile and the postnatal response to HCA. In this study, an immunoassay of 24 analytes customized to reflect the perinatal immune response was used to analyze profiles from umbilical cord and postnatal blood with placental histopathology to (1) characterize the intrauterine immune environment of preterm infants compared to term-born controls, and (2) test the hypothesis that exposure to histologic chorioamnionitis is associated with an altered immune and neurotrophic profile in the first week after very preterm birth.

\section{MATERIALS AND METHODS}

\section{Study Population}

Term (GA>37 weeks) and preterm (GA<33 weeks) infants were recruited to a longitudinal cohort study designed to investigate the effect of preterm birth on brain development, at the Royal Infirmary of Edinburgh, UK (30). Ethical approval was obtained from the UK National Research Ethics Service and parents provided written informed consent (South East Scotland Research Ethics Committee 16/SS/0154). For objective 1, we included infants if umbilical cord blood samples were available (59 preterm and 55 term), and for objective 2 we included preterm infants with placental histopathology and postnatal blood samples $(n=96)$.

\section{Dried Blood Spot Sample Analysis}

Dried blood spot samples (DBSS) were taken from the umbilical cord following delivery for both preterm cases and term-born controls. For preterm infants, an additional sample was collected on day 5 of life. A customized multiple sandwich immunoassay based on Meso-Scale technology was used to measure blood spot levels of Interleukin(IL)1- $\beta$, IL-2, IL-4, IL-5, IL-6, IL-8, IL-10, IL12p70, IL-17, IL-18, Monocyte chemotactic protein-1 (MCP-1), Macrophage inflammatory protein- $1 \alpha$ (MIP-1 $\alpha)$, Macrophage inflammatory protein-1 $\beta$ (MIP-1 $\beta$ ), Tumor necrosis factor- $\alpha$ (TNF- $\alpha$ ), Tumor necrosis factor- $\beta$ (TNF- $\beta$ ), Brain-derived neurotropic factor (BDNF), Granulocyte-macrophage colonystimulating factor (GM-CSF), Interferon- $\gamma$ (IFN- $\gamma)$, C-reactive protein (CRP), matrix-metalloproteinase 9 (MMP-9), Regulated 
upon activation, normal T cell expressed and secreted (RANTES) and Complement components C3, C5a and C9.

Two $3.2 \mathrm{~mm}$ disks from the DBSS were punched into each well of Nunc 96-well polystyrene microwell plates (\#277143, Thermo Fisher Scientific). $130 \mu \mathrm{l}$ extraction buffer (PBS containing 1\% BSA (Sigma Aldrich \#A4503), 0.5\% Tween-20 (\#8.22184.0500, Merck Millipore), and complete protease inhibitor cocktail (\#11836145001, Roche Diagnostics) were added to each well, and the samples were incubated for 1 hour at room temperature on a microwell shaker set at $900 \mathrm{rpm}$. The extracts were analysed using U-plex plates (Meso-Scale Diagnostics (MSD), Maryland, US) coated with antibodies specific for IL-1 $\beta$, IL-2, IL-4, IL-5, IL-6, IL-8, IL-12, IL-17, TNF- $\alpha$, MIP-1 $\beta$ on one plate (\#K15067 customized) and BDNF, GM-CSF, IL-10, IL-18, IFN- $\gamma$, TNF- $\beta$, MCP-1, MIP- $1 \alpha$ on another (\#K151AC customized) (both MSD). Supplier's instructions were followed, and extracts were analysed undiluted. A third multiplex analysis was developed in-house applying extracts diluted 1:10 in diluent 7 (\#R54BB, MSD) using antibodies specific for C3 (HYB030-07 and HYB030-06, SSI Antibodies, Copenhagen, Denmark), C5a (10604-MM04 and 10604-MM06, Sino Biological, Eschborn, Germany), C9 (Rplex kit \#F21XZ, MSD), MMP-9 (BAF911 and MAB911), RANTES (MAB278 and AF278NA) and CRP (BAM17072 and MAB1701) (all R\&D Systems, Minneapolis, US) for coating the U-plex plate and for detection, respectively. Coating antibodies (used at $1 \mu \mathrm{g} / \mathrm{mL}$, except CRP used at $10 \mathrm{ng} / \mathrm{mL}$ ) were biotinylated (using EZ-Link Sulfo-NHS-LC-Biotin \#21327, Thermo Fisher Scientific) in-house (if not already biotinylated at purchase) and detection antibodies were SULFO-tagged (R91AO, MSD), both at a challenge ratio of 20:1. The following calibrators were used: C3: \#PSP-109 (Nordic Biosite, Copenhagen, DK), C5a: \#10604-HNAE (Sino Biological), C9: \#F21XZ (from R-plex kit, MSD), MMP-9: \#911-MP, RANTES: \#278-RN and CRP: \#1707-CR/CF (all from R\&D Systems). Calibrators were diluted in diluent 7 , detection antibodies (used at $1 \mu \mathrm{g} / \mathrm{mL}$, except CRP used at $100 \mathrm{ng} / \mathrm{mL}$ ) were diluted in diluent 3 (\#R50AP, MSD). Controls were made in-house from part of the calibrator solution in one batch, aliquoted in portions for each plate and stored at $-20^{\circ} \mathrm{C}$ until use. The samples were prepared on the plates as recommended from the manufacturer and were immediately read on the QuickPlex SQ 120 (MSD). Analyte concentrations were calculated from the calibrator curves on each plate using 4PL logistic regression using the MSD Workbench software. Intra-assay variations were calculated from 16 measurements of a pool of the same control sample on the same plate. Inter-assay variations were calculated from controls analysed in duplicate on each plate during the sample analysis, 4 plates in total. Limits of detection were calculated as 2.5 standard deviations from duplicate measurements of the zero calibrator. The higher detection limit was defined as the highest calibrator concentration. Median intra-assay variation was $8.2 \%$ and median inter-assay variation was $11.1 \%$. Detection limits are detailed in Table $\mathbf{S} \mathbf{1}$.

\section{Placental Examination}

Placental examination was performed by an experienced perinatal pathologist (M.J.E.) and placental reaction patterns were reported according to the site of inflammation, using a structured system (31). HCA was defined as the presence of an inflammatory response in the placental membranes of any grade or stage. Maternal inflammatory response (MIR) was defined as the presence of chorionitis, chorioamnionitis or intervillositis. Fetal inflammatory response (FIR) was defined as the presence of vasculitis in the chorionic plate or funisitis involving any vessel of the umbilical cord.

\section{Statistical Analysis}

Participant demographics are described as mean \pm SD if normally distributed and mean (range) if skewed. Student's T test or Mann-Whitney $U$ were used to compare distributions, and Chi-square tests were used to compare proportions. Analytes with values less than the level of detection $(<$ LOD) were assigned the lowest detectable level prior to statistical analysis, and analytes with concentrations $<$ LOD in $\geq 75 \%$ of samples were excluded from subsequent statistical analysis. Data normality testing with Shapiro-Wilk confirmed a non-normal distribution of analyte concentrations, so to investigate group differences in blood immune mediator profiles we used the Mann-Whitney U or Kruskal Wallis and post hoc Dunn test, with Bonferroni correction for multiple tests.

Principal component analysis (PCA) was used to identify analytes contributing to variance in the cord blood profile and analytes that contributed to PCs with eigenvalues $>1$ were then entered as independent variables in a logistic regression model to predict preterm or term category. Analytes contributing to variability within PCs predictive of gestational category were then investigated individually using Spearman's rank order correlation to identify developmentally regulated analytes most strongly correlated with gestational age. Statistical analyses were performed using SPSS version 24.0 (IBM Corp., Armonk, NY), with the exception of PCA, which was performed using $\mathrm{R}$ version 3.6.1 (R Core Team, 2019).

\section{RESULTS}

\section{Umbilical Cord Blood Profile Associated With Preterm Birth}

Umbilical cord blood samples were available for 59 term and 55 preterm infants. Participant characteristics are detailed in Table 1. 10 analytes (GM-CSF, IFN- $\gamma$, IL-2, IL-4, IL-5, IL-10, IL-12, IL-17, MIP- $1 \alpha$ and TNF- $\beta$ ) were $<$ LOD in $\geq 75 \%$ of samples and were therefore excluded from subsequent analysis. Median and interquartile range of analytes are shown in Table 2. There were significant group differences for 9 immune mediators ( $\mathrm{p}<0.004$, Bonferroni corrected). Pro-inflammatory proteins IL-6, MCP-1 and CRP were elevated in the cord blood of preterm infants whilst BDNF, C3, C9, IL-18, MMP-9 and RANTES were decreased compared to controls born at term. PCA showed that 
TABLE 1 | Clinical characteristics of participants for umbilical cord blood analysis.

\begin{tabular}{|c|c|c|c|}
\hline & Preterm n = 55 & Term $\mathbf{n}=59$ & $p$-value \\
\hline Mean gestational age, weeks (range) & $29^{+0}\left(23^{+3}-32^{+0}\right)$ & $39^{+4}\left(37^{+3}-42^{+0}\right)$ & $<0.001$ \\
\hline Mean birthweight, g (range) & $1202(454-2110)$ & $3549(2556-4800)$ & $<0.001$ \\
\hline Mean birthweight z-score (SD) & $-0.2696(1.24)$ & $0.6828(1.08)$ & $<0.001$ \\
\hline Male sex, $\mathrm{n}(\%)$ & $33(60)$ & $30(51)$ & 0.326 \\
\hline \multicolumn{4}{|l|}{ Maternal factors } \\
\hline BMI, mean (SD) & $26.2(5.8)$ & $26.4(5.5)$ & 0.845 \\
\hline Pre-eclampsia, n (\%) & $3(5)$ & $3(5)$ & 0.930 \\
\hline Antenatal steroids, n (\%) & $54(98)$ & NA & NA \\
\hline Antenatal magnesium sulphate, n (\%) & $52(95)$ & NA & NA \\
\hline \multicolumn{4}{|l|}{ Delivery mode, $n(\%)$ : } \\
\hline Vaginal & $23(42)$ & $23(39)$ & 0.484 \\
\hline Caesarean & $32(58)$ & $36(61)$ & 0.223 \\
\hline -Pre-labor & $22(40)$ & $27(46)$ & 0.506 \\
\hline- In labor & $10(18)$ & $9(15)$ & \\
\hline Any labor, n (\%) & $33(60)$ & $32(54)$ & 0.535 \\
\hline Histologic chorioamnionitis, n (\%) & $24(44)$ & $10(17)$ & 0.002 \\
\hline $\mathrm{MIR}+\mathrm{FIR}-$ & 11 & 2 & \\
\hline $\mathrm{MIR}+\mathrm{FIR}+$ & 13 & 8 & \\
\hline
\end{tabular}

NA, Not applicable.

five principal components (eigenvalues $>1$ ) explained $76 \%$ of the variance in the cord blood profile with the majority of variance explained by the first two components (25\% and $20 \%$ respectively, Table S2). Projection of individual inflammatory profiles onto the first two principal components is shown in Figure 1.

In a logistic regression model to predict preterm or term category based on cord blood profile, principal components predicted gestational age category with a classification accuracy of $86 \%$ (95\% CI 0.78-0.92), p value $=1.242 \times 10^{-14}$ (Table 3). The percentage contribution of each analyte to the principal components is shown in Figure 2. Amongst immune mediators contributing to variability within the principal components that predicted gestational category, correlation analysis showed that cord blood MMP-9 and BDNF were highly correlated with gestational age at birth (rho $>0.65$, $\mathrm{p}<0.001$ ) (Table 4).

\section{Histologic Chorioamnionitis Is Associated With an Altered Immune Profile on Day 5 After Very Preterm Birth}

Ninety-six preterm infants had placental histopathology and postnatal day 5 blood samples. Participant characteristics are detailed in Table 5. Thirty-one infants (32\%) were exposed to HCA and 18 of those had histopathological evidence of a fetal inflammatory response. Infants with HCA exposure had lower GA at birth than infants without HCA: mean GA $28^{+2}$ weeks versus $29^{+4}$ weeks $(\mathrm{p}=0.03$ ), were more likely to have been delivered vaginally $(\mathrm{p}<0.001)$ and more likely to have prolonged rupture of membranes prior to delivery $(\mathrm{p}<0.001)$. However, there were no statistically significant group differences in exposure to antenatal steroids or magnesium sulphate, birthweight or infant sex.

The concentrations of eight analytes (BDNF, C3, C5a, C9, IL-8, MCP-1, MIP-1 $\beta$ and MMP-9) differed on day 5 in preterm

TABLE 2 | Cord blood analytes in preterm infants and term-born controls.

\begin{tabular}{|c|c|c|c|c|c|}
\hline \multirow[t]{2}{*}{ Analyte (pg/ml) } & \multicolumn{2}{|c|}{ Preterm $n=55$} & \multicolumn{2}{|c|}{ Term $\mathbf{n}=59$} & \multirow[t]{2}{*}{ p-value* } \\
\hline & Median & Q1, Q3 & Median & Q1, Q3 & \\
\hline BDNF & 22.27 & $6.04,36.90$ & 62.33 & $43.18,113.68$ & $<0.001$ \\
\hline C3 & 3081995.73 & $1858060.85,4569905.86$ & 4447458.83 & $3520957.75,5613382.51$ & $<0.001$ \\
\hline C5a & 3694.52 & $2420.56,11470.85$ & 7136.48 & $4059.83,9443.64$ & 0.004 \\
\hline C9 & 1236.73 & 215.00, 6901.99 & 12628.69 & $3608.60,43156.07$ & $<0.001$ \\
\hline CRP & 100.88 & $89.00,12054.79$ & 89.00 & $89.00,89.00$ & $<0.001$ \\
\hline $\mathrm{IL}-1 \beta$ & 0.26 & $0.26,0.49$ & 0.11 & $0.02,0.34$ & 0.016 \\
\hline IL-6 $6^{\star \star}$ & 0.45 & $0.45,6.00$ & 0.45 & $0.45,0.45$ & $<0.001$ \\
\hline IL-8 & 12.05 & $5.75,81.08$ & 8.29 & $4.89,14.71$ & 0.134 \\
\hline IL-18 & 25.62 & $10.35,38.34$ & 41.27 & $27.50,54.78$ & 0.003 \\
\hline MCP-1 & 109.09 & $68.69,210.75$ & 48.12 & $38.54,66.63$ & $<0.001$ \\
\hline MIP-1 $\beta$ & 12.01 & $7.47,18.82$ & 9.72 & $6.88,18.24$ & 0.331 \\
\hline MMP-9 & 14000.68 & $4485.50,40077.02$ & 155885.89 & $87194.67,358084.73$ & $<0.001$ \\
\hline RANTES & 1460.12 & $702.74,2754.37$ & 3127.55 & 1817.10, 5295.39 & $<0.001$ \\
\hline TNF- $\alpha$ & 0.27 & $0.27,0.37$ & 0.27 & $0.27,0.27$ & 0.304 \\
\hline
\end{tabular}

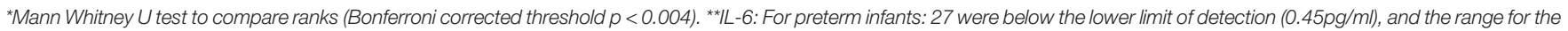
remaining 28 was $0.46-249.56 \mathrm{pg} / \mathrm{ml}$. For term infants: 50 were below the lower limit of detection and the range for the remaining 9 was $0.66-11.46 \mathrm{pg} / \mathrm{ml}$. 


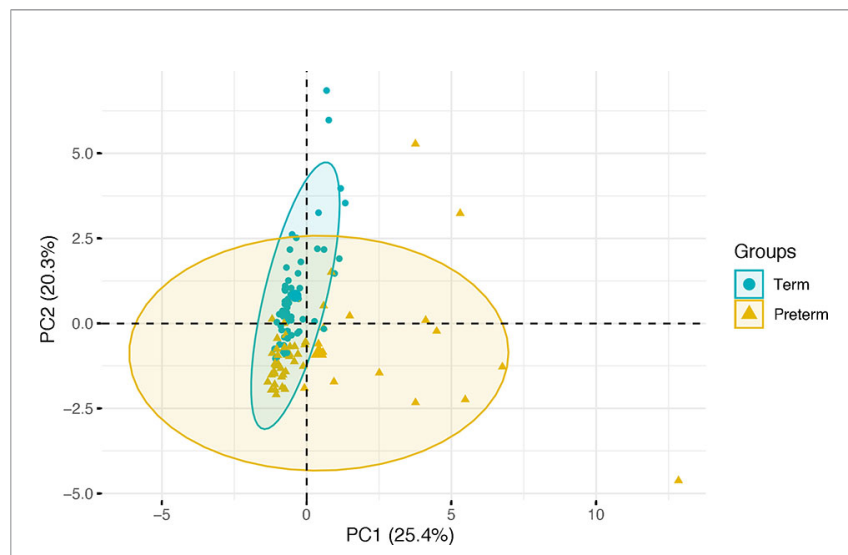

FIGURE 1 | Projection of individual cord blood inflammatory profiles onto the first two principal components, grouped by gestational age category. Term infants are represented in green and preterm infants in gold, with ellipses modelled on the mean and covariances of each group.

infants who were exposed to HCA compared to those who were not exposed (Mann Whitney $\mathrm{U}$ test, $\mathrm{p}<0.05$ ). These differences appear to be driven by histological evidence of a fetal inflammatory response for all but C5a (Kruskal Wallis with Dunn's post hoc test, $\mathrm{p}<0.05$ ) (Table 6).

\section{DISCUSSION}

By combining placental histopathology with a customized array of immune mediators in umbilical cord blood and postnatal blood from a large group of mother-infant dyads, this study characterizes differences in the systemic immune profile of very preterm infants compared with term-born controls, and demonstrates that exposure to HCA with evidence of a fetal inflammatory response is associated with postnatal immune dysregulation on day 5 after birth.

The umbilical cord blood immune profile was distinctly proinflammatory in preterm infants with significant elevations in proteins associated with the acute phase response: IL-6, MCP-1 and CRP. In contrast, six proteins were elevated in healthy termborn controls, suggesting developmental regulation with increasing gestational age: BDNF, C3, C9, IL-18, MMP-9 and RANTES. Eight proteins were increased on postnatal day 5 in preterm infants exposed to HCA compared to preterm infants without HCA: BDNF, C3, C5a, C9, IL-8, MCP-1, MIP-1 $\beta$ and MMP-9. Our findings are consistent with previous studies showing that the neonatal systemic

TABLE 3 | Logistic regression for the prediction of gestational age category using principal components derived from the umbilical cord blood profile.

\begin{tabular}{cccc}
\hline & B & $\boldsymbol{\beta}$ & p-value \\
\hline PC1 & 2.8189 & 5.3424 & 0.000337 \\
PC2 & -2.6162 & -4.4331 & $1.71 \mathrm{e}-06$ \\
PC3 & -0.1343 & -0.1850 & 0.648519 \\
PC4 & 0.8840 & 1.0084 & 0.135172 \\
PC5 & -0.8628 & -0.9076 & 0.027207
\end{tabular}

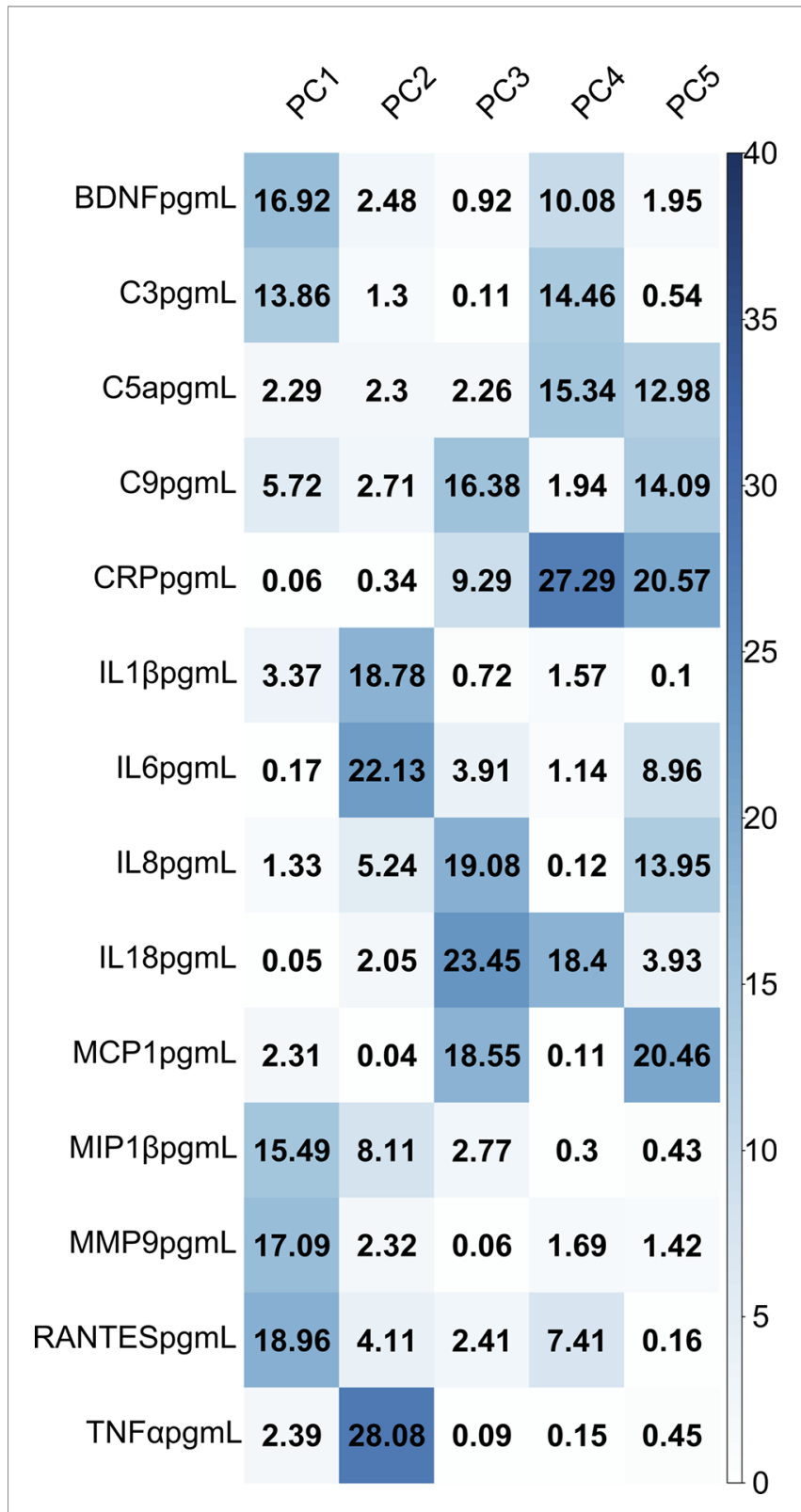

FIGURE 2 | Heatmap demonstrating the percentage contribution of each analyte to variability in the cord blood profile, grouped by principal component.

inflammatory response can be dysregulated and prolonged in the weeks after preterm birth $(4,27,28,32)$, but additionally suggest that this is programmed by a fetal inflammatory response.

BDNF expression has previously been shown to correlate with gestational age and postnatal age $(29,33)$ but here we show upregulation in preterm infants exposed to HCA with evidence of a fetal inflammatory response. BDNF belongs to the family of neurotrophins: an important group of signaling molecules responsible for neuronal growth, maturation and synaptic plasticity during development (34). Prematurity, placental dysfunction and fetal growth restriction have all been associated 
TABLE 4 | Correlation between individual analytes that contributed to the principal components predictive of gestational category and gestational age at birth.

\begin{tabular}{lcc}
\hline Analyte & Spearman's rho & p-value \\
\hline MMP-9 & 0.685 & $4.1162 \times 10^{-17}$ \\
BDNF & 0.654 & $2.8795 \times 10^{-15}$ \\
RANTES & 0.346 & 0.000160 \\
C3 & 0.290 & 0.002 \\
IL-1 $\beta$ & 0.190 & 0.043 \\
\hline
\end{tabular}

with reduced levels of $\operatorname{BDNF}(29,35-37)$ which may have important implications for long-term brain health. Reduced BDNF in the neonatal period has been associated with increased risk of developing autism spectrum disorder (38) whilst elevated BDNF in the weeks after preterm birth is associated with better cognitive performance in childhood $(39,40)$.

C3 was identified as a novel postnatal marker of exposure to intrauterine inflammation. The complement cascade plays a key role in the innate immune response (41) but is a potent inflammatory system which when dysregulated can cause significant tissue damage following injury. The complement cascade can be activated through several mechanisms, but all component pathways converge at Complement protein C3 (42). C3 participates in multiple key processes affecting developing brain architecture, including tagging of synapses for pruning by microglia $(43,44)$. The complement system is under-developed in preterm infants and complement regulators are low $(45,46)$, which may contribute to an uncontrolled complement response in the context of inflammation (47). We have previously shown that the downstream anaphylatoxin, C5a is elevated in the cerebrospinal fluid (CSF) of preterm infants when compared to term-born controls (6) and numerous studies beyond the neonatal period have also implicated complement C3 dysregulation in CNS pathology including neurodevelopmental disorders (48), multiple sclerosis (49), traumatic brain injury (50) and neurodegeneration (51).

MMP-9 on postnatal day 7 has previously been shown to correlate with the severity of funisitis following extremely preterm birth (27). MMP-9 is a member of the zinc-dependent endopeptidases that prototypically cleave extracellular matrix (ECM), cell adhesion molecules and cell surface receptors. Matrix-metalloproteinases also modulate the inflammatory response through the regulation of endothelial barrier function, cytokine activity and chemotactic gradient formation (52). The ECM is a key regulator of neural network development and plasticity through the stabilization of synaptic contacts. Dysregulation of MMP-9 during a critical window of CNS vulnerability may therefore have long-term consequences on structural connectivity (53). MMP-9 is higher in the CSF of preterm infants when compared to term-born controls and also higher amongst preterm infants with post hemorrhagic ventricular dilatation (PHVD) when compared to those without brain injury $(6,54)$. Elevated plasma MMP-9 is also associated with hypoxic-ischemic encephalopathy, correlating with severity of injury in human infants born at term (55-57).

The data provide new insights into immune dysregulation in the context of HCA and support several lines of evidence that suggest a link between HCA, immune response and neurodevelopment. First, activation of the fetal inflammatory response is an independent predictor of neonatal morbidity after adjustment for gestational age and obstetric indication for delivery (20). Second, sequencing studies have shown that exposure to HCA results in fetal immune programming in preterm infants with modulation of monocyte responses (5860). Third, intra-amniotic inflammation is associated with adverse perinatal outcomes whether or not microbes are detected (61). Fourth, exposure to HCA has been associated with an increased risk of intraventricular hemorrhage (62), white

TABLE 5 | Characteristics of preterm infants with day 5 blood samples.

\begin{tabular}{|c|c|c|c|}
\hline & No HCA $n=65$ & HCA $n=31$ & p-value \\
\hline Mean gestational age, weeks (range) & $29^{+4}\left(24^{+0}-32^{+0}\right)$ & $28^{+2}\left(23^{+2}-32^{+0}\right)$ & 0.030 \\
\hline Mean birthweight, g (range) & $1246(454-1915)$ & 1187 (500-2060) & 0.447 \\
\hline Mean birthweight z-score (SD) & $-0.3287(1.23)$ & $0.1870(0.69)$ & 0.010 \\
\hline Male sex, $\mathrm{n}(\%)$ & $37(57)$ & $18(58)$ & 0.916 \\
\hline Antenatal steroids, n (\%) & $61(94)$ & $30(97)$ & 0.546 \\
\hline Antenatal magnesium sulphate, n (\%) & $59(91)$ & $30(97)$ & 0.290 \\
\hline \multicolumn{4}{|l|}{ Delivery mode, $\mathrm{n}(\%)$ : } \\
\hline Vaginal & $15(23)$ & $23(74)$ & $<0.001$ \\
\hline Caesarean & $50(77)$ & $8(26)$ & 0.003 \\
\hline -Pre-labor & $34(68)$ & $8(100)$ & 0.033 \\
\hline -In labor & $16(32)$ & $0(0)$ & \\
\hline Any labor, n (\%) & $31(48)$ & $23(74)$ & 0.014 \\
\hline Prolonged rupture of membranes, $\mathrm{n}(\%)$ & $8(12)$ & $14(45)$ & $<0.001$ \\
\hline Early onset sepsis, n (\%) & $4(6)$ & $4(13)$ & 0.263 \\
\hline Late onset sepsis, n (\%) & $9(14)$ & $4(13)$ & 0.955 \\
\hline Bronchopulmonary dysplasia, n (\%) & $18(28)$ & $12(39)$ & 0.189 \\
\hline Necrotizing enterocolitis, n (\%) & $3(5)$ & $5(16)$ & 0.042 \\
\hline Retinopathy of prematurity, n (\%) & $3(5)$ & $5(16)$ & 0.042 \\
\hline
\end{tabular}

Prolonged rupture of membranes: $>24$ hours before delivery. Sepsis: Positive blood culture with a pathogenic organism and/or antibiotic treatment course for $\geq 5$ days. Early-onset sepsis: $<72$ hours after birth, late-onset sepsis: $>72$ hours after birth. Bronchopulmonary dysplasia: supplemental oxygen therapy or respiratory support at $36+{ }^{0}$ weeks gestational age. Necrotizing enterocolitis: medical treatment for $\geq 7$ days or surgical treatment. Retinopathy of prematurity: requiring treatment. 
TABLE 6 | Day 5 blood analyte concentrations for preterm infants exposed to histologic chorioamnionitis compared to those not exposed.

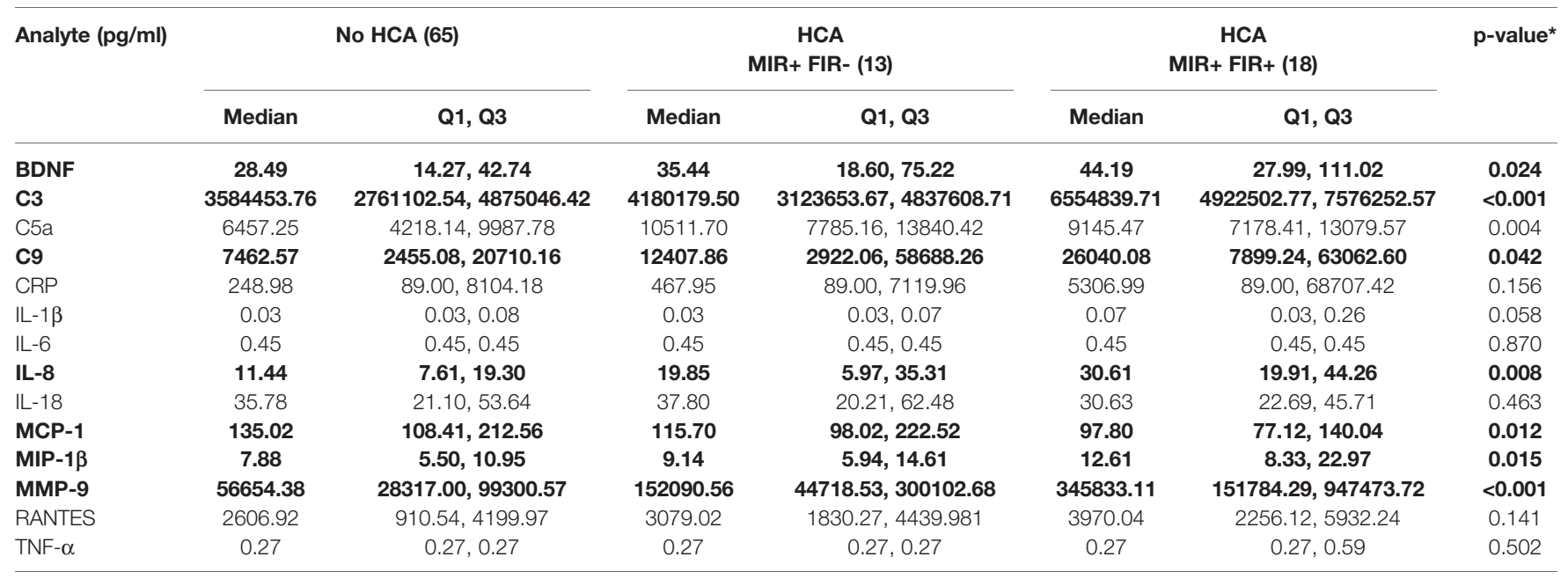

*Kruskal-Wallis test comparing three groups. Analytes highlighted in bold demonstrated significant group differences in a pairwise comparison of no HCA versus MIR+ FIR+ groups using post hoc Dunn's test. For C5a, post hoc tests showed the contrast was due to no HCA versus MIR+ FIR-.

matter injury $(17,18)$ and neurodevelopmental impairment $(15$, 63 ) in children who were delivered preterm. Finally, intrauterine infection or stimulation with lipopolysaccharide can induce a systemic fetal inflammatory response, neuroinflammation and white matter injury (64-66). It is estimated that 3-5\% of infants born by spontaneous vaginal delivery at term are exposed to HCA (67). Given that HCA is associated with cerebral palsy among children born at term (68), and maternal immune activation in term pregnancies is linked to neurodevelopmental and psychiatric diagnoses (69), further work is warranted to determine the relevance of our observations in preterm infants to births at term complicated by HCA.

Strengths of the study include investigation of a large number of inflammation-associated proteins representative of the perinatal immune response, and a data driven approach to characterize the inflammatory profile associated with very preterm birth and exposure to HCA. A limitation of the study is that the concentration of anti-inflammatory cytokines IL-4 and IL-10 were below the level of detection in our participants and so inferences about the balance of damaging and protective factors could not be explored. Another limitation is that amniotic fluid was not available for microbial analysis. Recent transcriptomic studies have shown that the presence of a fetal inflammatory response is more strongly associated with microbial invasion rather than sterile inflammation (70) but an alternative study design would be required to investigate differences in the postnatal immune profile of infants exposed to intra-amniotic infection compared to sterile inflammation.

Our data suggest that systemic fetal inflammation modulates neurotrophic capability and complement system activation in the perinatal period. However, a larger sample size would be required to perform sub-group analyses based on gestational age or sex, and to investigate possible confounding by antenatal steroids, mode of delivery and postnatal events.

Future work with a larger sample size and a replication cohort is needed to investigate the relationship between immune profiles and lung disease, gastrointestinal complications and neurocognitive outcomes following preterm birth, and to test for causality.

By combining placental histopathology with a comprehensive assessment of the immune response, we have shown that very preterm infants have a distinct pro-inflammatory profile in umbilical cord blood and that fetal inflammation is associated with an altered neonatal immune profile on postnatal day 5 . These results focus research attention on improved detection of fetuses exposed to intrauterine inflammation and suggest there may be a therapeutic window for targeted intervention that could reduce the risk of co-morbidities associated with HCA.

\section{DATA AVAILABILITY STATEMENT}

The raw data supporting the conclusions of this article will be made available by the authors, without undue reservation.

\section{ETHICS STATEMENT}

Ethical approval was obtained from the UK National Research Ethics Service and parents provided written informed consent (South East Scotland Research Ethics Committee 16/SS/0154).

\section{AUTHOR CONTRIBUTIONS}

GS conceived and designed the study, acquired and analyzed data, and drafted the article. PG and ME analyzed data and revised the article critically for important intellectual content. NB-L and KS acquired data, analysed data and revised the article critically for important intellectual content. DS and GL acquired data, and revised the article critically for important intellectual content. SC supervised acquisition of data and revised the article critically for important intellectual content. JB conceived and designed the study, supervised acquisition of data, analysed data 
and drafted the article. All authors contributed to the article and approved the submitted version.

\section{FUNDING}

Financial support for this study was provided by Theirworld (www.theirworld.org). This work was undertaken in the Medical Research Council Centre for Reproductive Health, which is funded by a Medical Research Council Centre grant (Medical Research Council G1002033).

\section{REFERENCES}

1. Al-Haddad BJS, Oler E, Armistead B, Elsayed NA, Weinberger DR, Bernier R, et al. The Fetal Origins of Mental Illness. Am J Obstetrics Gynecol (2019) 221 (6):549-62. doi: 10.1016/j.ajog.2019.06.013

2. Pape K, Tamouza R, Leboyer M, Zipp F. Immunoneuropsychiatry - Novel Perspectives on Brain Disorders. Nat Rev Neurol (2019) 15(6):317-28. doi: 10.1038/s41582-019-0174-4

3. Hagberg H, Mallard C, Ferriero DM, Vannucci SJ, Levison SW, Vexler ZS, et al. The Role of Inflammation in Perinatal Brain Injury. Nat Rev Neurol (2015) 11(4):192-208. doi: 10.1038/nrneurol.2015.13

4. Skogstrand K, Hougaard DM, Schendel DE, Bent NP, Svaerke C, Thorsen P. Association of Preterm Birth With Sustained Postnatal Inflammatory Response. Obstetr Gynecol (2008) 111(5):1118-28. doi: 10.1097/ AOG.0b013e31817057fb

5. Matoba N, Yu Y, Mestan K, Pearson C, Ortiz K, Porta N, et al. Differential Patterns of 27 Cord Blood Immune Biomarkers Across Gestational Age. Pediatrics (2009) 123(5):1320-8. doi: 10.1542/peds.2008-1222

6. Boardman JP, Ireland G, Sullivan G, Pataky R, Fleiss B, Gressens P, et al. The Cerebrospinal Fluid Inflammatory Response to Preterm Birth. Front Physiol (2018) 9:1299. doi: 10.3389/fphys.2018.01299

7. Romero R, Gómez R, Chaiworapongsa T, Conoscenti G, Kim JC, Kim YM. The Role of Infection in Preterm Labour and Delivery. Paediatr Perinatal Epidemiol (2001) 15 Suppl 2:41-56. doi: 10.1046/j.1365-3016.2001.00007.x

8. Goldenberg RL, Hauth JC, Andrews WW. Intrauterine Infection and Preterm Delivery. New Engl J Med (2000) 342(20):1500-7. doi: 10.1056/ NEJM200005183422007

9. Bose C, Laughon M, Allred EN, Van Marter LJ, O'Shea TM, Ehrenkranz RA, et al. Blood Protein Concentrations in the First Two Postnatal Weeks That Predict Bronchopulmonary Dysplasia Among Infants Born Before the 28th Week of Gestation. Pediatr Res (2011) 69(4):347-53. doi: 10.1203/ PDR.0b013e31820a58f3

10. Villamor-Martinez E, Álvarez-Fuente M, Ghazi AMT, Degraeuwe P, Zimmermann LJI, Kramer BW, et al. Association of Chorioamnionitis With Bronchopulmonary Dysplasia Among Preterm Infants: A Systematic Review, Meta-Analysis, and Metaregression. JAMA Netw Open (2019) 2(11): e1914611. doi: 10.1001/jamanetworkopen.2019.14611

11. Been JV, Lievense S, Zimmermann LJ, Kramer BW, Wolfs TG. Chorioamnionitis as a Risk Factor for Necrotizing Enterocolitis: A Systematic Review and MetaAnalysis. J Pediatr (2013) 162(2):236-42.e2. doi: 10.1016/j.jpeds.2012.07.012

12. Hofer N, Kothari R, Morris N, Müller W, Resch B. The Fetal Inflammatory Response Syndrome Is a Risk Factor for Morbidity in Preterm Neonates. Am J Obstetrics Gynecol (2013) 209(6):542.e1-.e11. doi: 10.1016/j.ajog.2013.08.030

13. Ozalkaya E, Karatekin G, Topcuoğlu S, Gürsoy T, Ovalı F. Morbidity in Preterm Infants With Fetal Inflammatory Response Syndrome. Pediatr International: Off J Japan Pediatr Soc (2016) 58(9):850-4. doi: 10.1111/ ped. 12895

14. Francis F, Bhat V, Mondal N, Adhisivam B, Jacob S, Dorairajan G, et al. Fetal Inflammatory Response Syndrome (FIRS) and Outcome of Preterm Neonates - a Prospective Analytical Study. J Maternal-Fetal Neonatal Med: Off J Eur Assoc Perinatal Med Fed Asia Oceania Perinatal Societies Int Soc Perinatal Obstet (2019) 32(3):488-92. doi: 10.1080/14767058.2017.1384458

\section{ACKNOWLEDGMENTS}

We are grateful to the families who consented to participate in the study.

\section{SUPPLEMENTARY MATERIAL}

The Supplementary Material for this article can be found online at: https://www.frontiersin.org/articles/10.3389/fimmu.2021.722489/ full\#supplementary-material

15. Venkatesh KK, Leviton A, Hecht JL, Joseph RM, Douglass LM, Frazier JA, et al. Histologic Chorioamnionitis and Risk of Neurodevelopmental Impairment at Age 10 Years Among Extremely Preterm Infants Born Before 28 Weeks of Gestation. Am J Obstetrics Gynecol (2020) 223 (5):745.e1-10. doi: 10.1016/j.ajog.2020.05.001

16. Shatrov JG, Birch SC, Lam LT, Quinlivan JA, McIntyre S, Mendz GL. Chorioamnionitis and Cerebral Palsy: A Meta-Analysis. Obstetr Gynecol (2010) 116(2 Pt 1):387-92. doi: 10.1097/AOG.0b013e3181e90046

17. Leviton A, Allred EN, Kuban KC, Hecht JL, Onderdonk AB, O'Shea TM, et al. Microbiologic and Histologic Characteristics of the Extremely Preterm Infant's Placenta Predict White Matter Damage and Later Cerebral Palsy. The ELGAN Study. Pediatr Res (2010) 67(1):95-101. doi: 10.1203/ PDR.0b013e3181bf5fab

18. Anblagan D, Pataky R, Evans MJ, Telford EJ, Serag A, Sparrow S, et al. Association Between Preterm Brain Injury and Exposure to Chorioamnionitis During Fetal Life. Sci Rep (2016) 6:37932. doi: 10.1038/srep37932

19. Sullivan G, Galdi P, Cabez MB, Borbye-Lorenzen N, Stoye DQ, Lamb GJ, et al. Interleukin-8 Dysregulation is Implicated in Brain Dysmaturation Following Preterm Birth. Brain Behav Immun (2020) 90:311-8. doi: 10.1016/ j.bbi.2020.09.007

20. Gomez R, Romero R, Ghezzi F, Yoon BH, Mazor M, Berry SM. The Fetal Inflammatory Response Syndrome. Am J Obstetrics Gynecol (1998) 179 (1):194-202. doi: 10.1016/S0002-9378(98)70272-8

21. Gotsch F, Romero R, Kusanovic JP, Mazaki-Tovi S, Pineles BL, Erez O, et al. The Fetal Inflammatory Response Syndrome. Clin Obstetrics Gynecol (2007) 50(3):652-83. doi: 10.1097/GRF.0b013e31811ebef6

22. Hecht JL, Fichorova RN, Tang VF, Allred EN, McElrath TF, Leviton A. Relationship Between Neonatal Blood Protein Concentrations and Placenta Histologic Characteristics in Extremely Low GA Newborns. Pediatr Res (2011) 69(1):68-73. doi: 10.1203/PDR.0b013e3181fed334

23. Døllner H, Vatten L, Halgunset J, Rahimipoor S, Austgulen R. Histologic Chorioamnionitis and Umbilical Serum Levels of Pro-Inflammatory Cytokines and Cytokine Inhibitors. BJOG: An Int J Obstetrics Gynaecol (2002) 109(5):534-9. doi: 10.1111/j.1471-0528.2002.01028.x

24. Mestan K, Yu Y, Thorsen P, Skogstrand K, Matoba N, Liu X, et al. Cord Blood Biomarkers of the Fetal Inflammatory Response. J Maternal-Fetal Neonatal Med: Off J Eur Assoc Perinatal Med Fed Asia Oceania Perinatal Societies Int Soc Perinatal Obstet (2009) 22(5):379-87. doi: 10.1080/14767050802609759

25. Armstrong-Wells J, Donnelly M, Post MD, Manco-Johnson MJ, Winn VD, Sebire G. Inflammatory Predictors of Neurologic Disability After Preterm Premature Rupture of Membranes. Am J Obstetrics Gynecol (2015) 212 (2):212.e1-9. doi: 10.1016/j.ajog.2014.09.016

26. Kacerovsky M, Cobo T, Andrys C, Musilova I, Drahosova M, Hornychova H, et al. The Fetal Inflammatory Response in Subgroups of Women With Preterm Prelabor Rupture of the Membranes. J Maternal-Fetal Neonatal Med: Off J Eur Assoc Perinatal Med Fed Asia Oceania Perinatal Societies Int Soc Perinatal Obstet (2013) 26(8):795-801. doi: 10.3109/ 14767058.2013.765404

27. Leviton A, Hecht JL, Allred EN, Yamamoto H, Fichorova RN, Dammann O. Persistence After Birth of Systemic Inflammation Associated With Umbilical Cord Inflammation. J Reprod Immunol (2011) 90(2):235-43. doi: 10.1016/ j.jri.2011.03.009 
28. Dammann O, Allred EN, Fichorova RN, Kuban K, O’Shea TM, Leviton A. Duration of Systemic Inflammation in the First Postnatal Month Among Infants Born Before the 28th Week of Gestation. Inflammation (2016) 39 (2):672-7. doi: 10.1007/s10753-015-0293-z

29. Leviton A, Allred EN, Yamamoto H, Fichorova RN, Kuban K, O’Shea TM, et al. Antecedents and Correlates of Blood Concentrations of Neurotrophic Growth Factors in Very Preterm Newborns. Cytokine (2017) 94:21-8. doi: 10.1016/j.cyto.2017.03.012

30. Boardman JP, Hall J, Thrippleton MJ, Reynolds RM, Bogaert D, Davidson DJ, et al. Impact of Preterm Birth on Brain Development and Long-Term Outcome: Protocol for a Cohort Study in Scotland. BMJ Open (2020) 10(3): e035854. doi: 10.1136/bmjopen-2019-035854

31. Khong TY, Mooney EE, Ariel I, Balmus NC, Boyd TK, Brundler MA, et al. Sampling and Definitions of Placental Lesions: Amsterdam Placental Workshop Group Consensus Statement. Arch Pathol Lab Med (2016) 140 (7):698-713. doi: 10.5858/arpa.2015-0225-CC

32. Liston A, Carr EJ, Linterman MA. Shaping Variation in the Human Immune System. Trends Immunol (2016) 37(10):637-46. doi: 10.1016/j.it.2016.08.002

33. Rao R, Mashburn CB, Mao J, Wadhwa N, Smith GM, Desai NS. Brain-Derived Neurotrophic Factor in Infants $<32$ Weeks Gestational Age: Correlation With Antenatal Factors and Postnatal Outcomes. Pediatr Res (2009) 65(5):548-52. doi: 10.1203/PDR.0b013e31819d9ea5

34. Huang EJ, Reichardt LF. Neurotrophins: Roles in Neuronal Development and Function. Annu Rev Neurosci (2001) 24:677-736. doi: 10.1146/ annurev.neuro.24.1.677

35. Matoba N, Ouyang F, Mestan KK, Porta NF, Pearson CM, Ortiz KM, et al. Cord Blood Immune Biomarkers in Small for Gestational Age Births. J Dev Origins Health Dis (2011) 2(2):89-98. doi: 10.1017/S2040174411000018

36. D'Angelo A, Ceccanti M, Petrella C, Greco A, Tirassa P, Rosso P, et al. Role of Neurotrophins in Pregnancy, Delivery and Postpartum. Eur J Obstetrics Gynecol Reprod Biol (2020) 247:32-41. doi: 10.1016/j.ejogrb.2020.01.046

37. Flöck A, Weber SK, Ferrari N, Fietz C, Graf C, Fimmers R, et al. Determinants of Brain-Derived Neurotrophic Factor (BDNF) in Umbilical Cord and Maternal Serum. Psychoneuroendocrinology (2016) 63:191-7. doi: 10.1016/ j.psyneuen.2015.09.028

38. Skogstrand K, Hagen CM, Borbye-Lorenzen N, Christiansen M, BybjergGrauholm J, Baekvad-Hansen M, et al. Reduced Neonatal Brain-Derived Neurotrophic Factor is Associated With Autism Spectrum Disorders. Trans Psychiatry (2019) 9(1):252. doi: 10.1038/s41398-019-0587-2

39. Kuban KCK, Heeren T, O'Shea TM, Joseph RM, Fichorova RN, Douglass L, et al. Among Children Born Extremely Preterm a Higher Level of Circulating Neurotrophins Is Associated With Lower Risk of Cognitive Impairment at School Age. J Pediatr (2018) 201:40-8.e4. doi: 10.1016/j.jpeds.2018.05.021

40. Leviton A, Joseph RM, Fichorova RN, Allred EN, Gerry Taylor H, Michael O'Shea T, et al. Executive Dysfunction Early Postnatal Biomarkers Among Children Born Extremely Preterm. J Neuroimmune Pharmacol: Off J Soc Neuro Immune Pharmacol (2019) 14(2):188-99. doi: 10.1007/s11481-0189804-7

41. Merle NS, Noe R, Halbwachs-Mecarelli L, Fremeaux-Bacchi V, Roumenina LT. Complement System Part II: Role in Immunity. Front Immunol (2015) 6 (257):257. doi: 10.3389/fimmu.2015.00257

42. Merle NS, Church SE, Fremeaux-Bacchi V, Roumenina LT. Complement System Part I - Molecular Mechanisms of Activation and Regulation. Front Immunol (2015) 6:262. doi: 10.3389/fimmu.2015.00262

43. Schafer DP, Lehrman EK, Kautzman AG, Koyama R, Mardinly AR, Yamasaki R, et al. Microglia Sculpt Postnatal Neural Circuits in an Activity and Complement-Dependent Manner. Neuron (2012) 74(4):691-705. doi: 10.1016/j.neuron.2012.03.026

44. Gorelik A, Sapir T, Ben-Reuven L , Reiner O. Complement C3 Affects Rac1 Activity in the Developing Brain. Front Mol Neurosci (2018) 11:150. doi: $10.3389 /$ fnmol.2018.00150

45. McGreal EP, Hearne K, Spiller OB. Off to a Slow Start: Under-Development of the Complement System in Term Newborns is More Substantial Following Premature Birth. Immunobiology (2012) 217(2):176-86. doi: 10.1016/ j.imbio.2011.07.027

46. Grumach AS, Ceccon ME, Rutz R, Fertig A, Kirschfink M. Complement Profile in Neonates of Different Gestational Ages. Scandinavian J Immunol (2014) 79(4):276-81. doi: 10.1111/sji.12154
47. Fragopoulou AF, Qian Y, Heijtz RD, Forssberg H. Can Neonatal Systemic Inflammation and Hypoxia Yield a Cerebral Palsy-Like Phenotype in Periadolescent Mice? Mol Neurobiol (2019) 56(10):6883-900. doi: 10.1007/ s12035-019-1548-8

48. Magdalon J, Mansur F, Teles e Silva AL, de Goes VA, Reiner O, Sertié AL. Complement System in Brain Architecture and Neurodevelopmental Disorders. Front Neurosci (2020) 14:23. doi: 10.3389/fnins.2020.00023

49. Werneburg S, Jung J, Kunjamma RB, Ha SK, Luciano NJ, Willis CM, et al. Targeted Complement Inhibition at Synapses Prevents Microglial Synaptic Engulfment and Synapse Loss in Demyelinating Disease. Immunity (2020) 52 (1):167-82.e7. doi: 10.1016/j.immuni.2019.12.004

50. Alawieh A, Langley EF, Weber S, Adkins D, Tomlinson S. Identifying the Role of Complement in Triggering Neuroinflammation After Traumatic Brain Injury. J Neurosci: Off J Soc Neurosci (2018) 38(10):2519-32. doi: 10.1523/ JNEUROSCI.2197-17.2018

51. Wu T, Dejanovic B, Gandham VD, Gogineni A, Edmonds R, Schauer S, et al. Complement C3 Is Activated in Human $\mathrm{AD}$ Brain and Is Required for Neurodegeneration in Mouse Models of Amyloidosis and Tauopathy. Cell Rep (2019) 28(8):2111-23.e6. doi: 10.1016/j.celrep.2019.07.060

52. Fingleton B. Matrix Metalloproteinases as Regulators of Inflammatory Processes. Biochim Biophys Acta (BBA) Mol Cell Res (2017) 1864(11, Part A):2036-42. doi: 10.1016/j.bbamcr.2017.05.010

53. Reinhard SM, Razak K, Ethell IM. A Delicate Balance: Role of MMP-9 in Brain Development and Pathophysiology of Neurodevelopmental Disorders. Front Cell Neurosci (2015) 9:280. doi: 10.3389/fncel.2015.00280

54. Okamoto T, Takahashi S, Nakamura E, Nagaya K, Hayashi T, Shirai M, et al. Increased Expression of Matrix Metalloproteinase- 9 and Hepatocyte Growth Factor in the Cerebrospinal Fluid of Infants With Posthemorrhagic Hydrocephalus. Early Hum Dev (2010) 86(4):251-4. doi: 10.1016/ j.earlhumdev.2010.03.007

55. Walsh BH, Boylan GB, Kenny LC, Dempsey EM, Murray DM, Neonatal Brain Research G. Targeted Biomarker Discovery in Hypoxic-Ischemic Encephalopathy; Correlation With Early Continuous EEG. Pediatr Res (2011) 70(5):134-. doi: 10.1038/pr.2011.359

56. Bednarek N, Svedin P, Garnotel R, Favrais G, Loron G, Schwendiman L, et al Increased MMP-9 and TIMP-1 in Mouse Neonatal Brain and Plasma and in Human Neonatal Plasma After Hypoxia-Ischemia: A Potential Marker of Neonatal Encephalopathy. Pediatr Res (2012) 71(1):63-70. doi: 10.1038/ pr.2011.3

57. Savard A, Brochu M-E, Chevin M, Guiraut C, Grbic D, Sébire G. Neuronal Self-Injury Mediated by IL-1 $\beta$ and MMP-9 in a Cerebral Palsy Model of Severe Neonatal Encephalopathy Induced by Immune Activation Plus Hypoxia-Ischemia. J Neuroinflamm (2015) 12(1):111. doi: 10.1186/s12974015-0330-8

58. de Jong E, Hancock DG, Wells C, Richmond P, Simmer K, Burgner D, et al. Exposure to Chorioamnionitis Alters the Monocyte Transcriptional Response to the Neonatal Pathogen Staphylococcus Epidermidis. Immunol Cell Biol (2018) 96(8):792-804. doi: 10.1111/imcb.12037

59. Bermick J, Gallagher K, denDekker A, Kunkel S, Lukacs N, Schaller M. Chorioamnionitis Exposure Remodels the Unique Histone Modification Landscape of Neonatal Monocytes and Alters the Expression of Immune Pathway Genes. FEBS J (2019) 286(1):82-109. doi: 10.1111/febs.14728

60. Sabic D, Koenig JM. A Perfect Storm: Fetal Inflammation and the Developing Immune System. Pediatr Res (2020) 87(2):319-26. doi: 10.1038/s41390-0190582-6

61. Combs CA, Gravett M, Garite TJ, Hickok DE, Lapidus J, Porreco R, et al. Amniotic Fluid Infection, Inflammation, and Colonization in Preterm Labor With Intact Membranes. Am J Obstetrics Gynecol (2014) 210(2):125.e1-.e15. doi: 10.1016/j.ajog.2013.11.032

62. Villamor-Martinez E, Fumagalli M, Mohammed Rahim O, Passera S, Cavallaro G, Degraeuwe P, et al. Chorioamnionitis Is a Risk Factor for Intraventricular Hemorrhage in Preterm Infants: A Systematic Review and Meta-Analysis. Front Physiol (2018) 9:1253. doi: 10.3389/fphys.2018.01253

63. Salas AA, Faye-Petersen OM, Sims B, Peralta-Carcelen M, Reilly SD, McGwin GJr., et al. Histological Characteristics of the Fetal Inflammatory Response Associated With Neurodevelopmental Impairment and Death in Extremely Preterm Infants. J Pediatr (2013) 163(3):652-7.e1-2. doi: 10.1016/ j.jpeds.2013.03.081 
64. Hagberg H, Peebles D, Mallard C. Models of White Matter Injury: Comparison of Infectious, Hypoxic-Ischemic, and Excitotoxic Insults. Ment Retard Dev Disabil Res Rev (2002) 8(1):30-8. doi: 10.1002/mrdd.10007

65. Burd I, Balakrishnan B, Kannan S. Models of Fetal Brain Injury, Intrauterine Inflammation, and Preterm Birth. Am J Reprod Immunol (N Y NY: 1989) (2012) 67(4):287-94. doi: 10.1111/j.1600-0897.2012.01110.x

66. Gussenhoven R, Westerlaken RJJ, Ophelders DRMG, Jobe AH, Kemp MW, Kallapur SG, et al. Chorioamnionitis, Neuroinflammation, and Injury: Timing is Key in the Preterm Ovine Fetus. J Neuroinflamm (2018) 15(1):113. doi: 10.1186/s12974-018-1149-x

67. Kim CJ, Romero R, Chaemsaithong P, Chaiyasit N, Yoon BH, Kim YM. Acute Chorioamnionitis and Funisitis: Definition, Pathologic Features, and Clinical Significance. Am J Obstetrics Gynecol (2015) 213(4 Suppl):S29-52. doi: 10.1016/j.ajog.2015.08.040

68. Shi Z, Ma L, Luo K, Bajaj M, Chawla S, Natarajan G, et al. Chorioamnionitis in the Development of Cerebral Palsy: A Meta-Analysis and Systematic Review. Pediatrics (2017) 139(6):e20163781. doi: 10.1542/peds.2016-3781

69. Estes ML, McAllister AK. Maternal Immune Activation: Implications for Neuropsychiatric Disorders. Sci (N Y NY) (2016) 353(6301):772-7. doi: 10.1126/science.aag3194

70. Motomura K, Romero R, Galaz J, Tarca AL, Done B, Xu Y, et al. RNA Sequencing Reveals Distinct Immune Responses in the Chorioamniotic
Membranes of Women With Preterm Labor and Microbial or Sterile IntraAmniotic Inflammation. Infect Immun (2021) 89(5):e00819-20. doi: 10.1128/ IAI.00819-20

Conflict of Interest: The authors declare that the research was conducted in the absence of any commercial or financial relationships that could be construed as a potential conflict of interest.

Publisher's Note: All claims expressed in this article are solely those of the authors and do not necessarily represent those of their affiliated organizations, or those of the publisher, the editors and the reviewers. Any product that may be evaluated in this article, or claim that may be made by its manufacturer, is not guaranteed or endorsed by the publisher.

Copyright (C) 2021 Sullivan, Galdi, Borbye-Lorenzen, Stoye, Lamb, Evans, Skogstrand, Chandran and Boardman. This is an open-access article distributed under the terms of the Creative Commons Attribution License (CC BY). The use, distribution or reproduction in other forums is permitted, provided the original author(s) and the copyright owner(s) are credited and that the original publication in this journal is cited, in accordance with accepted academic practice. No use, distribution or reproduction is permitted which does not comply with these terms. 